\title{
Glossaire Autophagie
}

Autophagie sélective : autophagie qui implique la séquestration d'un seul type de structure ou d'organite (par exemple, la mitochondrie: on parle de mitophagie; les gouttelettes lipidiques: on parle alors de lipophagie) qui dépend d'un récepteur autophagique (comme les protéines p62, NDP52 [nuclear domain 10 protein 52], optineurine) reconnu par une protéine de la famille ATG8 en général.

Autophagosome: vacuole autophagique initiale. Elle est formée d'une double membrane lipidique après extension du phagophore et contient du matériel cytoplasmique intact.

Autophagolysosome : vésicule résultant de la fusion entre l'autophagosome et les lysosomes, ultime étape de l'autophagie.

ATG : autophagy-related gene. Famille de gènes impliqués dans les processus autophagiques.

BCL2L13 : Bcl2 like protein 13. Induit la fragmentation mitochondriale et la mitophagie.

BNIP3 : Bcl2 interacting protein 3. En réponse aux dommages mitochondriaux, participe à la mitophagie.

Cargos : composants de la cellule (lipides, protéines) pris en charge par le processus autophagique.

CMA : chaperone-mediated autophagy ou autophagie relayée par des protéines chaperonnes (protéines aidant d'autres protéines pour leur maturation et pour prendre leur conformation correcte).

DFCP1 : double FYVE-domain containing protein 1. DFCPl est une protéine liant le phosphatidylinositol 3-phosphate (PI3P). Elle est un marqueur de l'omégasome (voir Omégasome).

FIP200 : focal adhesion kinase (FAK) family kinase-interacting protein of $200 \mathrm{kDa}$.

Ferritinophagie : mécanisme autophagique impliqué dans le renouvellement de la ferritine.

GABARAP : gamma-aminobutyric acid receptor-associated protein. Protéine impliquée dans les derniers stades de maturation de l'autophagosome.

LAMP2A : lysosome-associated membrane protein type 2A. Protéine lysosomale spécifique.

LC3 ou MAP1LC3 : microtubule-associated protein IA/1B-light chain 3. La forme cytosolique de LC3 (LC3-I) se conjugue à la phosphatidyléthanolamine pour former LC3-II recrutée à la membrane de l'autophagosome.

LIR : LC3-interacting region. Motif reconnu par LC3 permettant le recrutement vers l'autophagosome.

Lysosome : vésicule cytoplasmique acide contenant les enzymes nécessaires à la dégradation de tous les types de macromolécules. $\varepsilon l l e$ est commune à l'autophagie et à l'endo-phagocytose.

Mitophagie : autophagie sélective impliquée dans le contrôle des mitochondries.

mTORC1 : mammalian target of rapamycin complex 1. Régule négativement l'autophagie en phosphorylant ULK1.

Omégasome : protrusion du réticulum endoplasmique qui est à l'origine du phagophore. L'omegasome réunit les différentes protéines autophagiques et les lipides qui initient le processus de formation du phagophore.

Phagophore : formation membranaire issue du réticulum endoplasmique après constitution de l'omegasome. Il est à l'origine de l'autophagosome.

PI3KCIII : class III phosphoinositide 3-kinase. La phosphoinositide 3-kinase de classe III associée à VPS34 phosphoryle le phosphatidylinositol (PI) pour générer le phosphatidylinositol 3-phosphate (PI3P).

p62 : ou SQSTM1, sequestosome-1. Aussi appelée ubiquitin-binding protein p62, intervient dans la reconnaissance des protéines ubiquitinylées. UBD : ubiquitin-binding domain.

Ubiquitinylation ou ubiquitination : modification post-traductionnelle aboutissant à la fixation covalente d'une ou de plusieurs protéines d'ubiquitine sur une ou plusieurs lysines acceptrices de la protéine ciblée. Ces modifications biochimiques ont plusieurs fonctions. Elles permettent l'adressage de la protéine ubiquitinée (ou ubiquitinylée) vers le protéasome, le tri intracellulaire ou encore la reconnaissance par la protéine LC3 lors de l'autophagie sélective.

ULK1 : Unc-51-like autophagy-activating kinase 1. Kinase activée par sa phosphorylation par AMPK (AMP-activated protein kinase) et régulant négativement le complexe mTORCl.

UPR : unfolded protein response, réponse de la cellule permettant l'élimination des protéines dont la conformation est incorrecte.

VMP1 : vacuole membrane protein 1. Joue un rôle dans les étapes initiales de l'autophagie en se liant à bécline-1.

VPS34 : vacuolar protein sorting 34. Participe au complexe phosphoinositide 3-kinase. Phosphoryle le phosphatidylinositol.

WIPI : WD repeat domain phosphoinositide-interacting protein. Protéine autophagosomale dont l'action se situe entre celles de LC3 et ULKI.

Xénophagie : processus autophagique participant à l'élimination des bactéries et des virus.

ZFYVE1 : zinc finger FyVE-type containing 1. Intervient dans la liaison du phosphatidylinositol 3-phosphate.

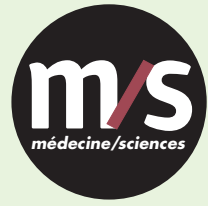

Tarifs d'abonnement $\mathrm{m} / \mathrm{s}-2017$

\section{Abonnez-vous}

à médecine/sciences
$>$ Grâce à $m / s$, vivez en direct les progrès des sciences biologiques et médicales

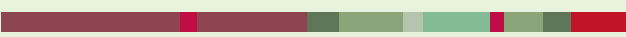

$$
\begin{gathered}
\text { Bulletin d'abonnement } \\
\text { page } 362 \text { dans ce numéro de } \mathrm{m} / \mathrm{s}
\end{gathered}
$$

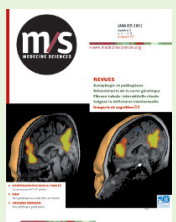

